\title{
Pediatric intracranial arteriovenous malformations: a single-center experience
}

\author{
Melissa A. LoPresti, MD,,2 Vijay M. Ravindra, MD, MSPH,, Monika Pyarali, BS,, \\ Eric Goethe, MD, ${ }^{1,2}$ Nisha Gadgil, MD, ${ }^{1,2}$ Kathyrn Wagner, MD, ${ }^{1,2}$ Peter Kan, MD, MPH, ${ }^{1,2}$ and \\ Sandi Lam, MD, MBA ${ }^{1,2}$

\begin{abstract}
'Division of Neurosurgery, Texas Children's Hospital, Houston; and 2Department of Neurosurgery, Baylor College of Medicine, Houston, Texas
\end{abstract}

\begin{abstract}
OBJECTIVE Intracranial arteriovenous malformations (AVMs) are a common cause of intracranial hemorrhage in children. Exploring outcomes of treatment and factors that predict recurrence may help guide the management of pediatric AVMs. In this study, the authors review a single-center experience with pediatric AVMs, aiming to identify factors contributing to recurrence and areas for improvement in treatment.
\end{abstract}

METHODS A single-center retrospective review of patients ages 0-18 years with AVMs in the period from 2005 to 2018 was conducted. Demographic, clinical, and radiographic data were collected and examined to compare the clinical and functional outcomes of patients managed with and those managed without open microsurgical resection.

RESULTS One hundred five patients were diagnosed and treated during the study period. Fifty (47.6\%) had been treated surgically and $35(33.3 \%)$ nonsurgically, and $20(19 \%)$ had been treated primarily at an outside institution and thus were excluded from further analysis. Of the 50 treated surgically, $92 \%$ had complete obliteration on postoperative imaging, with the remaining patients having residual AVM and undergoing re-resection or SRS, ultimately resulting in a $96 \%$ radiographic cure rate. The surgically treated patients were followed up for an average of 3.34 years. Eighty-two percent had a modified Rankin Scale (mRS) score of $0-2$. There was a recurrence rate of $12 \%$, with the time to recurrence averaging 327.86 days (range $85-596$ days). Of the 35 patients managed without open surgery, $77 \%$ underwent serial imaging surveillance, $20 \%$ underwent endovascular embolization, and $3 \%$ underwent stereotactic radiosurgery. Radiographic cure was seen in $6 \%$ of those treated nonsurgically, and all had undergone endovascular treatment alone. The nonsurgically treated patients were followed up for an average of 2.14 years. Eighty percent had an mRS score of 0-2.

CONCLUSIONS The data revealed that patients with lower-grade AVMs treated surgically and those with higher-grade AVMs managed without surgery have good functional and clinical outcomes. A high rate of recurrence was noted, and the possible contributing factors were explored. The authors encourage further study into mechanisms to reduce AVM recurrence in pediatric patients and look forward to advances in the medical management of lesions that are considered unresectable.

https://thejns.org/doi/abs/10.3171/2019.9.PEDS19235

KEYWORDS cerebral arteriovenous malformations; pediatrics; recurrence; outcomes; vascular disorders

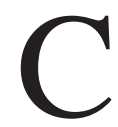

EREBRAL arteriovenous malformations (AVMs) are lesions of abnormal vascular connections that occur in the brain in approximately $10-18$ cases per 100,000 people. ${ }^{21}$ AVMs can remain asymptomatic for years or can lead to seizures or rupture, which can result in devastating intracranial hemorrhage and life-long disability. Ruptured AVMs account for a much higher proportion of spontaneous intracranial hemorrhages in pediatric patients than in adults, that is, $30 \%-50 \%$ compared to
$1.4 \%-2 \%$, respectively. ${ }^{7}$ With untreated AVMs, the annual risk of rupture in children is $4.4 \%-5.5 \%$, and that risk is significantly reduced after treatment. ${ }^{7.9}$ This reduction supports the role of early intervention in the management of pediatric intracranial AVMs.

Current methods for managing intracranial AVMs include resection, endovascular embolization, radiosurgery, or a combination of these modalities. ${ }^{7,911,12}$ While surgery remains the gold-standard therapy for immediate angio-

ABBREVIATIONS AVM = arteriovenous malformation; $\mathrm{mRS}$ = modified Rankin Scale; SRS = stereotactic radiosurgery.

SUBMITTED April 26, 2019. ACCEPTED September 10, 2019.

INCLUDE WHEN CITING Published online November 22, 2019; DOI: 10.3171/2019.9.PEDS19235. 
graphic resolution of low-grade intracranial AVMs, many high-grade lesions are not amenable to this therapeutic modality because of their high-risk features or eloquent location. ${ }^{7,9,26}$ Endovascular therapy via embolization can be used to treat or reduce the flow of large AVMs in multiple stages, aiming for cure or rendering the lesion more amenable to surgery or radiosurgery. Complete radiographic resolution following embolization therapy alone has been estimated to occur in $10 \%-40 \%$ of AVMs treated endovascularly., ${ }^{4,29}$ Additionally, stereotactic radiosurgery (SRS) is another primary or adjunctive treatment method with a $60 \%-70 \%$ obliteration rate ${ }^{6}$ after involution, which occurs over a period of at least 12-24 months following treatment. ${ }^{30}$ The SRS cure rate is highly dependent on lesion size; thus, SRS is typically used for small or residual lesions located in surgically inaccessible locations.

While these three modalities can be used independently, they can be also used in combination to provide the best chance of complete obliteration of the AVM. Multimodal treatment is largely dependent on the characteristics of the $\mathrm{AVM}$ and is therefore individualized based on parameters such as lesion size, feeding vessels, and location. ${ }^{7,9,11,12}$

We aimed to examine the Texas Children's Hospital experience with pediatric intracranial AVMs to highlight the use of individualized multimodal treatments and their outcomes in this population. We present our institutional experience with pediatric ruptured and unruptured AVMs across 13 years. We describe treatment using various therapies with the aim to better understand clinical and functional outcomes in pediatric AVMs treated surgically or nonsurgically. Additionally, we aim to identify which factors may contribute to AVM recurrence in this pediatric patient population and to single out areas for improvement in treatment modalities and options for patients with limited conventional treatments. We hypothesize that outcomes in this cohort are favorable across treatment modalities and that there are unique characteristics of AVMs that contribute to recurrence.

\section{Methods}

A retrospective chart review of all patients who had been seen and treated for intracranial AVMs at Texas Children's Hospital in the period from 2005 to 2018 was conducted with institutional review board approval. Subjects were included if they had a radiographic or pathological diagnosis of intracranial AVM, were younger than 18 years old, and had been seen at our institution for conservative treatment, endovascular treatment, radiosurgery, or surgical management of an AVM. Any subjects who had undergone primary treatment of any type at an outside facility prior to transfer to Texas Children's Hospital for further care were excluded from this study. Clinical and radiographic variables collected included demographic and descriptive data regarding AVM characteristics and treatment. Primary outcomes were AVM obliteration, recurrence, and functional outcomes as assessed using the modified Rankin Scale (mRS) for neurological disabilities at the last follow-up.

Data were analyzed using IBM SPSS Statistics version 26 (IBM Corp.). Comparisons were made using chi- square or Fisher's exact tests for categorical variables and independent-samples t-tests or one-way ANOVA for continuous variables. Correlations were assessed using Pearson's $r$ test. Results were considered significant at the $p<$ 0.05 level; all comparisons were two-tailed.

\section{Results}

Of the 105 patients identified at our institution, 50 had undergone surgical treatment and 35 had undergone nonsurgical treatment defined as serial surveillance, radiosurgery, or endovascular therapy alone. Twenty patients had received some or all of their treatment at external facilities and had been transferred to our institution for further management; these patients were excluded from this study.

The surgical group was older than the nonsurgical group, with a mean age of 9.82 years compared to 4.09 years $(\mathrm{p}=0.63$; Table 1$)$. The groups were both predominantly Hispanic with similar sex distributions. The nonsurgical group had a higher rate of concomitant AVMs or AVM syndromes, including hereditary hemorrhagic telangiectasia and Wyburn-Mason syndrome, than the nonsurgical group, though the difference was not statistically significant $(p=0.11)$. There was a wide range of locations and sizes of AVMs among both groups; however, lesions treated surgically were more commonly located in the frontal, temporal, and parietal lobes, whereas lesions in the nonsurgical group were located more commonly in the deep structures, including the basal ganglia, thalamus, brainstem, corpus callosum, or intraventricular region $(\mathrm{p}=$ $0.01)$. The average nidus size was larger in the nonsurgical group, $3.95 \mathrm{~cm}$ compared to $2.42 \mathrm{~cm}$ in the surgical group $(\mathrm{p}=0.01)$. Additionally, the nonsurgical group more commonly had lesions in eloquent locations $(\mathrm{p}=0.01)$, with deep venous drainage $(\mathrm{p}=0.01)$, with higher SpetzlerMartin $^{27}(p=0.01)$ or Lawton-Young ${ }^{16}(p=0.01)$ grades, with a diffuse nidus $(\mathrm{p}=0.01)$, and with more feeding arteries to the lesion $(p=0.01)$.

Seventy-two percent of those treated surgically had presented with rupture, and the most common presenting symptom in this group was headache (60\%; Table 2). Patients in the nonsurgical group also most commonly presented with headache $(49 \%)$ or had lesions that were incidentally found (32\%). Presenting symptoms had occurred for a similar duration in the two groups $(\mathrm{p}=0.11)$.

The outcomes of those with surgically treated AVMs are listed in Table 3. Thirty-two percent of these patients had undergone preoperative embolization, with a median of 1 embolization procedure (range 1-4) and a $12.5 \%$ complete obliteration rate after preoperative embolization. Following resection, this group had a $92 \%$ complete resection rate on immediate postoperative imaging; $8 \%$ were found to have residual AVM after surgery. Of the 4 patients with residual AVM, one underwent radiosurgery for treatment of the residual lesion; the other 3 patients underwent repeat surgery, with one having preoperative embolization prior to the repeat resection. Two underwent re-resection within a week of the initial resection, and the patient who underwent re-resection in a delayed fashion had incomplete medical records, so the reason for 
TABLE 1. Descriptive characteristics by treatment group

\begin{tabular}{|c|c|c|c|}
\hline Variable & Surgical & Nonsurgical & p Value \\
\hline No. of patients & 50 & 35 & \\
\hline \multicolumn{4}{|l|}{ Age in yrs } \\
\hline Mean & 9.82 & 4.09 & $0.63^{*}$ \\
\hline Range & $2-17$ & $1-17$ & \\
\hline Sex $(\%)$ & & & $0.84 \dagger$ \\
\hline Male & 52 & 54 & \\
\hline Female & 48 & 46 & \\
\hline Race/ethnicity (\%) & & & $0.23 \ddagger$ \\
\hline White & 40 & 26 & \\
\hline African American & 4 & 6 & \\
\hline Hispanic & 56 & 60 & \\
\hline Asian & 0 & 6 & \\
\hline Other/unknown & 0 & 3 & \\
\hline Presence of other AVMs or AVM syndromes & & & $0.11 \dagger$ \\
\hline Hereditary hemorrhagic telangiectasia & 4 & 3 & $0.29 \ddagger$ \\
\hline Wyburn-Mason syndrome & 0 & 3 & \\
\hline History of AVM in other location & 0 & 6 & \\
\hline Concern for syndrome, undiagnosed & 0 & 3 & \\
\hline \multicolumn{4}{|l|}{ AVM characteristics } \\
\hline Laterality (\%) & & & $0.11 \dagger$ \\
\hline $\mathrm{Rt}$ & 58 & 51 & \\
\hline Lt & 42 & 40 & \\
\hline Bilat & 0 & 9 & \\
\hline Lobe (\%) & & & $0.01 \ddagger$ \\
\hline Frontal & 40 & 20 & \\
\hline Temporal & 24 & 26 & \\
\hline Parietal & 26 & 9 & \\
\hline Occipital & 8 & 14 & \\
\hline Holohemispheric & 0 & 9 & \\
\hline Deep§ & 0 & 43 & \\
\hline Cerebellum & 12 & 3 & \\
\hline \multicolumn{4}{|l|}{ Size of nidus } \\
\hline Mean in $\mathrm{cm}$ & 2 & 4 & $0.01^{*}$ \\
\hline $0-3 \mathrm{~cm}(\%)$ & 70 & 29 & $0.01 \dagger$ \\
\hline $3-6 \mathrm{~cm}(\%)$ & 20 & 49 & \\
\hline$>6 \mathrm{~cm}(\%)$ & 4 & 20 & \\
\hline Unknown (\%) & 6 & 3 & \\
\hline Eloquent location (\%) & 24 & 69 & $0.01 \dagger$ \\
\hline Deep venous drainage (\%) & 24 & 69 & $0.01 \dagger$ \\
\hline SM grade $(\%)$ & & & $0.01 \ddagger$ \\
\hline I & 38 & 9 & \\
\hline II & 40 & 17 & \\
\hline III & 6 & 20 & \\
\hline IV & 8 & 37 & \\
\hline V & 0 & 14 & \\
\hline Unknown & 8 & 3 & \\
\hline Diffuse nidus (\%) & 36 & 80 & $0.01 \dagger$ \\
\hline
\end{tabular}


» CONTINUED FROM PAGE 153

TABLE 1. Descriptive characteristics by treatment group

\begin{tabular}{lccc}
\hline \multicolumn{1}{c}{ Variable } & Surgical & Nonsurgical & $p$ Value \\
\hline AVM characteristics (continued) & & & $\mathbf{0 . 0 1 \ddagger}$ \\
\hline LY grade (\%) & & 9 & \\
\hline I & 46 & 31 & \\
\hline II & 40 & 57 & $0.01^{*}$ \\
\hline II & 8 & 3 & $0.23 \dagger$ \\
\hline Unknown & 6 & 2.21 & $0.64 \dagger$ \\
\hline Signs of prior hemorrhage (\%) & 1.43 & 3 & $0.45 \dagger$ \\
\hline Presence of intranidal aneurysm (\%) & 12 & 26 & 11 \\
\hline Venous outlet obstruction (\%) & 30 & 6 & \\
\hline
\end{tabular}

$\mathrm{LY}=$ Lawton-Young; $\mathrm{SM}=$ Spetzler-Martin.

Boldface type indicates statistical significance.

${ }^{*} p$ value derived from independent samples t-test.

$\dagger p$ value derived from Fisher's exact test.

$\ddagger p$ value derived from chi-square test.

$\S$ Deep is defined as a location in the basal ganglia, thalamus, brainstem, corpus callosum, or intraventricular region.

the delayed re-resection is unknown, but it occurred 14 months after the initial resection. All 4 patients with residual AVM had complete obliteration on the most recent follow-up imaging. The surgical patients were followed up for an average of 3.34 years. A recurrence rate of $12 \%$ was noted in this group, with the time to recurrence averaging 327.86 days (range 85-596 days). Recurrence was defined as the interval development of AVM after postoperative imaging had demonstrated no appreciable residual AVM. Of the 6 patients with AVM recurrence, one had two recurrence episodes. Of the 7 episodes of recurrence, 6 were treated with redo craniotomy for AVM resection with one patient undergoing radiosurgery for treatment of the recurrence. All but 2 patients had complete obliteration of the recurrent AVM on the most recent follow-up imaging. Of the 50 patients treated surgically, $96 \%$ had radio-

TABLE 2. Clinical presentation of patients by treatment group

\begin{tabular}{lccc}
\hline \multicolumn{1}{c}{ Variable } & Surgical & Nonsurgical & p Value \\
\hline No. of patients & 50 & 35 & \\
\hline Rupture (\%) & 72 & 29 & $0.01^{*}$ \\
\hline Headache (\%) & 60 & 49 & $0.08^{*}$ \\
\hline Weakness (\%) & 14 & 17 & $0.84^{*}$ \\
\hline Speech disturbances (\%) & 2 & 11 & $1.00 \dagger$ \\
\hline Visual disturbances (\%) & 4 & 11 & $0.39 \dagger$ \\
\hline Imbalance (\%) & 20 & 3 & $0.44 \dagger$ \\
\hline Dysphagia (\%) & 0 & 11 & - \\
\hline Incidental finding (\%) & 12 & 32 & $0.02 \dagger$ \\
\hline Mean duration of symptoms in wks & 6.01 & 19.68 & $0.11 \ddagger$ \\
\hline
\end{tabular}

Boldface type indicates statistical significance.

${ }^{*} p$ value derived from chi-square test.

$\dagger p$ value derived from Fisher's exact test.

$\ddagger p$ value derived from paired-samples t-test. graphic cure at the last follow-up. The 2 patients who did not have radiographic cure had recurrent AVM; one was treated with radiosurgery, with a small residual nidus followed conservatively, and the other patient was followed up conservatively for a new small recurrence after having undergone two redo craniotomies for resection of the recurrent AVM. The decision for conservative management was based on shared decision-making with the families. Looking more closely at the group with recurrence, we noted that the patients were predominantly males of various ethnic and racial backgrounds, more commonly presented with rupture, had frontal or temporal nidus locations, and their nidi had one feeding artery, were typically smaller than $3 \mathrm{~cm}$ in size, were not typically deep or eloquently located, and were more diffuse. While the number of recurrences was too small to detect statistically significant differences, there were some subjective commonalities among the patients, namely nidus location, size, and diffusivity.

Postoperative complications included neurological deficits, wound complications, and hydrocephalus or CSF leakage. Neurological deficits in the surgically treated group included hemiparesis, ataxia, cranial neuropathies, cognitive impairment, speech delay, and visual field deficits. Eighty-two percent of the surgically treated patients had an mRS score of 0-2 at the last follow-up, indicating functional independence.

The outcomes of patients in the nonsurgical group are listed in Table 4. Seventy-seven percent underwent serial surveillance, $20 \%$ underwent endovascular embolization, and 3\% underwent SRS. Among those who underwent embolization, the median number of embolization procedures was 3 (range 1-6) with a complete obliteration rate of $14 \%$ after embolization alone. Complications of embolization included AVM rupture or delayed hemorrhage, hydrocephalus, and neurological deficits. There was no difference in the overall complication rate between the 
TABLE 3. Surgical group AVM outcomes

\begin{tabular}{lc}
\hline \multicolumn{1}{c}{ Variable } & Value \\
\hline Preop embolization (\%) & 32 \\
\hline Median no. of embolization procedures (range) & $1(1-4)$ \\
\hline Complete obliteration after embolization (\%) & 12.50 \\
\hline Complete resection postoperatively (\%) & 92 \\
\hline Postop complications (\%) & 10 \\
\hline Neurological deficit & 4 \\
\hline Hydrocephalus, CSF leakage, pseudomeningocele & 4 \\
\hline Wound complication* & 8 \\
\hline Residual AVM (\%)† & 12 \\
\hline Recurrent AVM (\%)† & \\
\hline Time to recurrence in days & $85-596$ \\
\hline Range & 327.86 \\
\hline Mean & 34 \\
\hline Additional procedures (\%) & 24 \\
\hline Preop EVD placement & 8 \\
\hline Preop decompressive craniectomy & 8 \\
\hline Hematoma evacuation & 6 \\
\hline Other (wound revision, ICP monitor) & 82 \\
\hline Length of FU in yrs & $0-11.75$ \\
\hline Range & 3.34 \\
\hline Mean & 96 \\
\hline Radiographic cure at FU (\%) & \\
\hline Functional outcome (\%) & \\
\hline Functionally independent (mRS score 0-2) & \\
\hline Functionally dependent (mRS score 3-6) & \\
\hline Unknown mRS score & \\
\hline
\end{tabular}

$E V D=$ external ventricular drain; FU = follow-up; ICP = intracranial pressure. * Wound complication is defined as wound infection, wound dehiscence, or bone flap resorption.

† Residual AVM was defined by the presence of residual AVM on postoperative imaging. Recurrent AVM was defined by the interval development of AVM after postoperative imaging demonstrated no residual AVM.

$\ddagger$ One patient had two episodes of recurrence, and that patient is counted here once, but each episode's time to recurrence is included.

two treatment groups $(\mathrm{p}<0.65)$. Neurological deficits in the nonsurgical group included hemiparesis, cognitive impairment, speech delay, and visual field deficits. None of the patients who experienced complete obliteration with embolization had a recurrence in the follow-up period. The nonsurgical patients were followed up for an average of 2.14 years. Radiographic cure was seen in $6 \%$ of the nonsurgical group, and all had undergone endovascular treatment alone. There were higher rates of radiographic cure in the surgical group than in the nonsurgical group ( $96 \%$ vs $6 \%, \mathrm{p}<0.01$ ). Delayed hemorrhage or AVM rupture was seen in $6 \%$ of the nonsurgical group throughout the follow-up. At the most recent follow-up, $80 \%$ of the patients in the nonsurgical group had an mRS score of $0-2$, indicating functional independence. There was no difference in the last mRS score $(\mathrm{p}<0.11)$ or functional independence $(\mathrm{p}<1.00)$ between the treatment groups.
TABLE 4. Nonsurgical group AVM outcomes

\begin{tabular}{|c|c|}
\hline Variable & Value \\
\hline \multicolumn{2}{|l|}{ Treatment modality (\%) } \\
\hline Endovascular embolization & 20 \\
\hline SRS & 3 \\
\hline Serial surveillance & 77 \\
\hline \multicolumn{2}{|l|}{ Embolization $(n=7)$} \\
\hline Median no. of embolization procedures (range) & $3(1-6)$ \\
\hline Complete obliteration after embolization (\%) & 14 \\
\hline \multicolumn{2}{|l|}{ Complications (\%) } \\
\hline AVM rupture or delayed hemorrhage & 6 \\
\hline Hydrocephalus, CSF leakage, pseudomeningocele & 6 \\
\hline Neurological deficit & 3 \\
\hline \multicolumn{2}{|l|}{ Additional procedures (\%) } \\
\hline EVD placement & 17 \\
\hline Decompressive craniectomy & 6 \\
\hline Hematoma evacuation & 3 \\
\hline Other (wound revision, ICP monitor, VPS) & 9 \\
\hline \multicolumn{2}{|l|}{ Length of FU in yrs } \\
\hline Range & $0-12$ \\
\hline Mean & 2.14 \\
\hline Radiographic cure at FU (\%) & 6 \\
\hline \multicolumn{2}{|l|}{ Functional outcome (\%) } \\
\hline Functionally independent (mRS score 0-2) & 80 \\
\hline Functionally dependent (mRS score 3-6) & 6 \\
\hline Unknown mRS score & 14 \\
\hline
\end{tabular}

$\mathrm{n}=$ number; VPS = ventriculoperitoneal shunt.

\section{Discussion}

This study represents a large series of pediatric intracranial AVMs. We found an overall radiographic cure of $96 \%$ for those treated surgically and $6 \%$ for those in the nonsurgical group. These rates are comparable to those quoted in the literature and underscore the success and benefit of surgical intervention.

We characterized functional outcomes using the mRS, with a score of 0-2 indicating functional independence. Both groups, surgical and nonsurgical, had good neurological functional outcomes, with $82 \%$ and $80 \%$, respectively, having $\mathrm{mRS}$ scores of $0-2$. This outcome corresponds with those in previous reports supporting improvement after treatment ${ }^{31}$ and indicates that an overwhelming majority of pediatric patients have functional independence after treatment, whether surgical or nonsurgical, with good outcomes.

\section{AVM Recurrence: When, How, and Why?}

Most notably, we found a recurrence rate of $12 \%$ among those who had undergone resection, with the time to recurrence averaging roughly 12 months; no recurrence was seen in the $6 \%$ of patients cured with nonsurgical treatment. A recent systematic review of 14 case reports and 16 case series examined the risk factors and timing for recurrence of resected brain AVMs, finding a $2.7 \%$ rate of 
recurrence in adult cases and a 9.5\%-14\% rate of recurrence in pediatric cases, with an average time to recurrence of 4.2 years. ${ }^{26}$ Lang et al. assessed 28 pediatric patients treated with AVM resection and noted a recurrence in $14.3 \%$ of them, occurring 50-60 weeks after the initial resection, with the recurrence risk identified as 0.07 per person-year. ${ }^{15}$ Interestingly, these authors also correlated a lower compactness score with AVM recurrence. ${ }^{15}$ Looking more closely at the group that experienced a recurrence in our study, we noted that most had a diffuse nidus as well, although the number of recurrences was not sufficiently powered to demonstrate statistical significance. Therefore, it is possible that diffuse AVMs may have a higher propensity for recurrence, or perhaps there was an occult residual lesion that could not be visualized on the immediate postoperative angiogram, as opposed to a true recurrence.

Given the relatively high rate of recurrence after complete resection, a deeper understanding of why this occurs and how to minimize recurrence is imperative. Among the AVMs that did recur, we found a higher prevalence in males and among patients who had presented with AVM rupture, had frontal or temporal nidus locations, had one nidus-feeding artery, had an AVM smaller than $3 \mathrm{~cm}$ in size, and had a more diffuse nidus. None of the patients with residual AVMs had a recurrence, although one patient had a recurrence twice. While these results bear no statistical significance, subjective findings may help guide future study, and comparisons with the literature can help inform management. A systematic review revealed that AVM recurrence in pediatric populations varies across a wide range of time, from 3 months to 17 years postresection, with an average time to recurrence of 4.82 years. ${ }^{14}$ Authors of this review also stratified recurrence according to whether or not patients underwent active follow-up imaging and found an earlier rate of detection among those undergoing surveillance, with a time to recurrence of 3.56 compared to 8.86 years. Moreover, those who did not have imaging follow-up had a higher risk of presenting with AVM rupture. These findings highlight the need for longterm follow-up and serial surveillance in pediatric patients with a history of intracranial AVM, with clear roles for detecting clinical and radiographic changes and critically evaluating conservative management versus treatment at each follow-up.

The literature on AVMs in adults reports a recurrence rate of approximately $2 \%-4 \% .^{13,26}$ The reason for the higher rate of recurrence among the pediatric AVM population is not fully understood, although multiple theories have been postulated. The concept of early postoperative changes hiding residual AVM which later presents as a recurrence is one such theory. ${ }^{22}$ Other authors have proposed that there are different angiogenic attributes of pediatric AVMs that foster vascular proliferation and subsequent recurrence. ${ }^{25}$ Additionally, the angioarchitecture of pediatric AVMs differs from that of adult AVMs, with exclusive deep venous drainage higher in pediatric populations and deep venous drainage associated with AVM recurrence. ${ }^{1,2,10}$ Moreover, some pediatric AVMs have a more diffuse architecture, perhaps predisposing to occult areas, which can foster a future recurrence. ${ }^{15}$ Going forward, we aim to understand the attributes of pediatric AVMs that lead to increased recurrences by looking at tissue pathology and genetic markers as possible means to elucidate this pathology and pathophysiology and perhaps open new avenues of treatment in the years to come. We also look forward to future large-volume studies sufficiently powered to explore associations with recurrences to further guide treatment.

\section{The Role of SRS}

One treatment modality used in a limited fashion in our study was SRS. This procedure was developed by Leskell in 1951 to destroy lesions inaccessible via open surgery by delivering beams of radiation to a designated brain area. ${ }^{17}$ It was first applied to intracranial AVMs in $1972 .{ }^{28}$ Currently, SRS remains one of the mainstays of primary and adjunctive therapy for inaccessible AVMs. SRS is most effective in treating small AVMs $\left(<3 \mathrm{~cm}\right.$ or $<10 \mathrm{~cm}^{3}$ in volume). ${ }^{6}$ The mechanism by which SRS causes AVM involution remains unclear, but it is thought to be similar to the formation of an atherosclerotic plaque wherein damage to the surrounding vessel walls stimulates proliferation of smooth muscle cells, which leads to narrowing and eventual occlusion of the vessel. ${ }^{30}$ Relatedly, the resolution of AVMs treated with SRS is delayed by several months to years, with complete obliteration seen at a minimum of 12-18 months in multiple adult series. ${ }^{6}$ Despite the delay in AVM resolution after SRS treatment, many studies support the use of SRS in pediatric AVMs, touting good obliteration rates of $60 \%-95 \% .{ }^{20,24}$ After treatment with an average radiation dose of $20 \mathrm{~Gy}$ and stratification by AVM size, obliteration rates of $80 \%$ in AVM lesions smaller than $3 \mathrm{ml}$ and $64.7 \%$ in lesions $3-10 \mathrm{ml}$ were seen. ${ }^{18}$

Our clinical practice favors the resection of all accessible lesions in noneloquent areas. For lesions located in deep, eloquent, or inaccessible areas of the brain, conservative treatment with serial surveillance is favored $(77 \%$ of nonsurgically treated patients in our study underwent imaging surveillance). Very few patients underwent embolization alone, and even fewer underwent SRS. Endovascular embolization of complex AVMs can change flow dynamics, increasing the rupture risk if the lesion is not completely embolized. ${ }^{5}$ Radiosurgery can help target deep remnants of the AVM adjacent to structures such as the internal capsule and thalamus. ${ }^{23}$ If the AVM is only partially obliterated with radiosurgery, studies have suggested that the rupture risk may be similar to the natural history of $2.6 \%$ per year. ${ }^{8}$ Additionally, in ARUBA (A Randomized Trial of Unruptured Brain Arteriovenous Malformations), which compared the risk of death and symptomatic stroke in adult patients with unruptured cerebral AVMs randomly assigned to medical management or medical management plus intervention, including neurosurgical, endovascular, or radiotherapy procedures, researchers found a significantly lower risk of death and neurological disability at 30 months' follow-up in the medical management group (15.1\% vs $46.2 \%) .{ }^{19}$ While the ARUBA findings are not generalizable to pediatric populations, based on an extrapolation of its findings and for the reasons aforementioned, we propose serial surveillance as a viable option in patients with high-grade unresectable lesions. 


\section{Additional Future Directions}

The nonsurgical cohort in our study comprised mostly patients with deep, diffuse, large, eloquent high-grade lesions with more feeding arteries, venous outflow obstruction, and deep venous drainage. This cohort was also associated with a higher proportion of multiple AVMs or AVM-associated syndromes. Despite a low cure rate in this group (6\% compared to $96 \%$ ), our study demonstrated comparable functional and clinical outcomes in the patients treated nonsurgically and surgically. We recognize that the majority of patients in the nonsurgical cohort were treated with serial surveillance, which inherently will not lead to a cure, unless spontaneous regression occurs. While our findings underscore the importance of patient selection when determining treatment modality, we also believe that these findings highlight a subset of pediatric patients with high-grade, unresectable AVMs that would benefit from further study to delineate alternative treatment modalities where conventional modalities offer limited benefit. We hope for advances in the medical management of AVMs and look toward understanding the genetics, molecular biology, and immunohistopathology of these lesions to find targets for medical treatments in the future.

\section{Study Limitations}

Limitations of this study include the small number of patients in each group and lack of power to assess statistical significance between smaller subgroups with stratification. With an increased sample size, more subtle differences between treatment modalities and clinical as well as functional outcomes could be better explored. Because of the low numbers of recurrences and residual lesions in our study, significant statistical analysis comparing associations with the rate of recurrence or residual lesions could not be performed. Given the retrospective study design, causality when assessing recurrence or residual lesions could not be assessed; prospective randomized control trials would be best to establish temporality and assess causality. Additionally, the single-institution retrospective design applied in our study reflects the practice variations of treating clinicians' choices, limiting our ability to assess, through a larger volume, patients treated using all types of modalities. Moreover, though the mRS is a validated scoring system commonly used to assess functional neurological outcomes, the scale is designed for adults and has not been validated in children, ${ }^{3}$ thereby making assessment of functional outcome challenging to definitively gauge in this patient population with the mRS score functioning as a proxy.

\section{Conclusions}

In sum, our study examined a sizable cohort of pediatric patients diagnosed with and treated for intracranial AVMs. We found that patients with lower-grade AVMs treated surgically and patients with higher-grade AVMs managed without surgery have good functional and clinical outcomes. We identified a high rate of recurrence and explored possible contributing factors. We hope that further examination of these factors and outcomes can better delineate the attributes of pediatric AVMs that lead to delayed and high rates of recurrence, and we look forward to future studies investigating the underlying reasons. We encourage further study into mechanisms to reduce AVM recurrence and look forward to advances in the medical management of lesions considered to be unresectable.

\section{References}

1. Aboukaïs R, Vinchon M, Quidet M, Bourgeois P, Leclerc X, Lejeune JP: Reappearance of arteriovenous malformations after complete resection of ruptured arteriovenous malformations: true recurrence or false-negative early postoperative imaging result? J Neurosurg 126:1088-1093, 2017

2. Andaluz N, Myseros JS, Sathi S, Crone KR, Tew JM Jr: Recurrence of cerebral arteriovenous malformations in children: report of two cases and review of the literature. Surg Neurol 62:324-331, 2004

3. Banks JL, Marotta CA: Outcomes validity and reliability of the modified Rankin scale: implications for stroke clinical trials: a literature review and synthesis. Stroke 38:10911096, 2007

4. Barr JC, Ogilvy CS: Selection of treatment modalities or observation of arteriovenous malformations. Neurosurg Clin $\mathbf{N}$ Am 23:63-75, 2012

5. Blauwblomme T, Bourgeois M, Meyer P, Puget S, Di Rocco F, Boddaert N, et al: Long-term outcome of 106 consecutive pediatric ruptured brain arteriovenous malformations after combined treatment. Stroke 45:1664-1671, 2014

6. Blount JP, Oakes WJ, Tubbs RS, Humphreys RP: History of surgery for cerebrovascular disease in children. Part III. Arteriovenous malformations. Neurosurg Focus 20(6):E11, 2006

7. Darsaut TE, Guzman R, Marcellus ML, Edwards MS, Tian L, Do HM, et al: Management of pediatric intracranial arteriovenous malformations: experience with multimodality therapy. Neurosurgery 69:540-556, 2011

8. El-Ghanem M, Kass-Hout T, Kass-Hout O, Alderazi YJ, Amuluru K, Al-Mufti F, et al: Arteriovenous malformations in the pediatric population: review of the existing literature. Intervent Neurol 5:218-225, 2016

9. Gross BA, Storey A, Orbach DB, Scott RM, Smith ER: Microsurgical treatment of arteriovenous malformations in pediatric patients: the Boston Children's Hospital experience. J Neurosurg Pediatr 15:71-77, 2015

10. Hetts SW, Cooke DL, Nelson J, Gupta N, Fullerton H, Amans $\mathrm{MR}$, et al: Influence of patient age on angioarchitecture of brain arteriovenous malformations. AJNR Am J Neuroradiol 35:1376-1380, 2014

11. Hoh BL, Ogilvy CS, Butler WE, Loeffler JS, Putman CM, Chapman PH: Multimodality treatment of nongalenic arteriovenous malformations in pediatric patients. Neurosurgery 47:346-358, 2000

12. Humphreys RP, Hoffman HJ, Drake JM, Rutka JT: Choices in the 1990s for the management of pediatric cerebral arteriovenous malformations. Pediatr Neurosurg 25:277-285, 1996

13. Ivanov AA, Alaraj A, Charbel FT, Aletich V, Amin-Hanjani S: Recurrence of cerebral arteriovenous malformations following resection in adults: does preoperative embolization increase the risk? Neurosurgery 78:562-571, 2016

14. Jimenez JE, Gersey ZC, Wagner J, Snelling B, Ambekar S, Peterson EC: Role of follow-up imaging after resection of brain arteriovenous malformations in pediatric patients: a systematic review of the literature. J Neurosurg Pediatr 19:149-156, 2017

15. Lang SS, Beslow LA, Bailey RL, Vossough A, Ekstrom J, Heuer GG, et al: Follow-up imaging to detect recurrence of surgically treated pediatric arteriovenous malformations. J Neurosurg Pediatr 9:497-504, 2012 
16. Lawton MT, Kim H, McCulloch CE, Mikhak B, Young WL: A supplementary grading scale for selecting patients with brain arteriovenous malformations for surgery. Neurosurgery 66:702-713, 2010

17. Leksell L: Stereotactic radiosurgery. J Neurol Neurosurg Psychiatry 46:797-803, 1983

18. Levy EI, Niranjan A, Thompson TP, Scarrow AM, Kondziolka D, Flickinger JC, et al: Radiosurgery for childhood intracranial arteriovenous malformations. Neurosurgery 47:834-842, 2000

19. Mohr JP, Parides MK, Stapf C, Moquete E, Moy CS, Overbey $\mathrm{JR}$, et al: Medical management with or without interventional therapy for unruptured brain arteriovenous malformations (ARUBA): a multicentre, non-blinded, randomised trial. Lancet 383:614-621, 2014

20. Nataf F, Ghossoub M, Schlienger M, Moussa R, Meder JF, Roux FX: Bleeding after radiosurgery for cerebral arteriovenous malformations. Neurosurgery 55:298-306, 2004

21. Novakovic RL, Lazzaro MA, Castonguay AC, Zaidat OO: The diagnosis and management of brain arteriovenous malformations. Neurol Clin 31:749-763, 2013

22. Pellettieri L, Svendsen P, Wikholm G, Carlsson CA: Hidden compartments in AVMs-a new concept. Acta Radiol 38:2-7, 1997

23. Potts MB, Young WL, Lawton MT: Deep arteriovenous malformations in the basal ganglia, thalamus, and insula: microsurgical management, techniques, and results. Neurosurgery 73:417-429, 2013

24. Shin M, Kawamoto S, Kurita H, Tago M, Sasaki T, Morita A, et al: Retrospective analysis of a 10-year experience of stereotactic radio surgery for arteriovenous malformations in children and adolescents. J Neurosurg 97:779-784, 2002

25. Sonstein WJ, Kader A, Michelsen WJ, Llena JF, Hirano A, Casper D: Expression of vascular endothelial growth factor in pediatric and adult cerebral arteriovenous malformations: an immunocytochemical study. J Neurosurg 85:838-845, 1996

26. Sorenson TJ, Brinjikji W, Bortolotti C, Kaufmann G, Lanzino G: Recurrent brain arteriovenous malformations (AVMs): a systematic review. World Neurosurg 116:e856-e866, 2018

27. Spetzler RF, Martin NA: A proposed grading system for arteriovenous malformations. J Neurosurg 65:476-483, 1986

28. Steiner L, Leksell L, Greitz T, Forster DM, Backlund EO: Stereotaxic radiosurgery for cerebral arteriovenous malformations. Report of a case. Acta Chir Scand 138:459-464, 1972
29. Strozyk D, Nogueira RG, Lavine SD: Endovascular treatment of intracranial arteriovenous malformation. Neurosurg Clin N Am 20:399-418, 2009

30. Tu J, Stoodley MA, Morgan MK, Storer KP: Responses of arteriovenous malformations to radiosurgery: ultrastructural changes. Neurosurgery 58:749-758, 2006

31. van Essen MJ, Han KS, Lo RTH, Woerdeman P, van der Zwan A, van Doormaal TPC: Functional and educational outcomes after treatment for intracranial arteriovenous malformations in children. Acta Neurochir (Wien) 160:21992205,2018

\section{Disclosures}

Dr. Kan is a consultant for Stryker and Cerenovus.

\section{Author Contributions}

Conception and design: Lam, LoPresti. Acquisition of data: LoPresti, Pyarali. Analysis and interpretation of data: LoPresti, Ravindra, Goethe. Drafting the article: LoPresti, Pyarali. Critically revising the article: Lam, LoPresti, Ravindra, Gadgil, Wagner, Kan. Reviewed submitted version of manuscript: all authors. Approved the final version of the manuscript on behalf of all authors: Lam. Statistical analysis: LoPresti, Goethe. Administrative/technical/material support: Lam, Kan. Study supervision: Lam, Ravindra, Kan.

\section{Supplemental Information Previous Presentations}

Portions of this work were presented at the AANS/CNS Section on Pediatric Neurological Surgery held in Nashville, TN, 2018.

\section{Correspondence}

Sandi Lam: Texas Children's Hospital, Baylor College of Medicine, Houston, TX. sklam@texaschildrens.org; sandi.lam@bcm. edu; sandilam@gmail.com. 